\title{
Preparation and characterization of cefuroxime axetil solid dispersions using hydrophilic carriers
}

\author{
Adinarayana Gorajana, Adhiyaman Rajendran, Lee Mun Yew, Kamal Dua ${ }^{1}$ \\ Department of Pharmaceutical Technology, Faculty of Medicine and Health Sciences, International Medical University, Bukit Jalil, Kuala \\ Lumpur 57000, Malaysia, ' $S c h o o l$ of Biomedical Sciences and Pharmacy, The University of Newcastle, NSW 2308, Australia
}

\begin{abstract}
Aim: The objective of the current study is to increase the dissolution rate of cefuroxime axetil (CA) by formation of binary CA solid dispersion using water soluble carriers such as polyvinylpyrrolidone (PVP K30) and polyethylene glycol (PEG 4000). Methods: Solid dispersions (SDs) between CA and PVP K30/PEG 4000 were formed by dissolving both compounds in a common solvent, methanol, which were rotary evaporated at $40^{\circ} \mathrm{C}$ for $12 \mathrm{~h}$. Physical mixtures between CA and PVP K30/PEG 4000 were also formulated as to compare the efficiency of SDs. The physicochemical properties of CA and all its formulations were then characterized using differential scanning calorimetric analysis (DSC), powder X-ray diffraction studies (PXRD), and Fourier transform infrared spectroscopy (FTIR). Results: All SD formulations were found to have a higher dissolution rate comparatively to pure CA, while only physical mixtures of PVP K30 were found having a significantly higher dissolution rate. The enhancement of dissolution rate SD by PVP K 30 may be caused by increase wettability, solubility, reduction in particle size or the formation of CA $\beta$ crystalline. Increment of dissolution rate of CA SDs by PEG 4000 similarly may be caused by increase wettability, solubility, and reduction in particle size. This phenomenon may also be caused by amorphization as suggested by DSC and PXRD. Conclusions: The SD of CA with PVP K30 and PEG 4000, lends an ample credence for better therapeutic efficacy.
\end{abstract}

Key words: Cefuroxime axetil, dissolution, polyethylene glycol 4000, polyvinylpyrrolidone K30, solubility

\section{INTRODUCTION}

Up to $40 \%$ of new chemical entities discovered by the pharmaceutical industry today are poorly soluble or lipophilic compounds. The solubility issues complicating the delivery of these new drugs also affect the delivery of many existing drugs. ${ }^{[1]}$ Poorly water-soluble drugs show unpredictable absorption, since their bioavailability depends upon dissolution in the gastrointestinal tract. ${ }^{[2-4]}$ The dissolution characteristics of poorly soluble drugs can be enhanced by several methods. ${ }^{[5-7]}$ Solid dispersion (SD) is one of the effective and widely used techniques for dissolution enhancement. ${ }^{[8]}$ The two basic procedures used to prepare SDs are the melting or

\section{Address for correspondence: \\ Dr. Adinarayana Gorajana, \\ Department of Pharmaceutical Technology, Faculty of Medicine and Health Sciences, International Medical University, Bukit Jalil, Kuala Lumpur, 57000, Malaysia. \\ E-mail: adinarayana_gorajana@imu.edu.my}

\begin{tabular}{|l|l|}
\hline \multicolumn{2}{|c|}{ Access this article online } \\
\hline Quick Response Code: & Website: \\
\hline & www.jpionline.org \\
\cline { 2 - 2 } & DOI: \\
\hline
\end{tabular}

fusion $^{[9]}$ and solvent evaporation ${ }^{[10]}$ techniques. The increase in dissolution rate for SDs can be attributed to a number of factors, ${ }^{[1]}$ which include reduction in particle size, absence of aggregation or agglomeration of fine crystallites of the drug, possible solubilization effect of the polymer, excellent wettability, and dispersibility of the drug from SD and partial conversion of the drug into amorphous form.

Cefuroxime (CF), a semi-synthetic broad-spectrum $2^{\text {nd }}$ generation cephalosporin, is among one of the antibiotics widely used to treat bacterial infections. ${ }^{[12]}$ Besides treating lower respiratory tract infections, CF may also be used to treat upper respiratory tract infections, skin and soft tissue infections, and genitourinary tract infections caused by susceptible bacteria. ${ }^{[12]}$ It may also be employed for prophylaxis purposes in conditions such as coronary artery bypass grafting surgery or elective cholecystectomy. ${ }^{[13]}$

Despite usage only. To enable oral intake, CF may be given as an acetyloxyethyl ester, cefuroxime axetil (CA), which is a prodrug. ${ }^{[12]}$ This prodrug form when taken orally shows a bioavailability of $30 \%$ while fasting and $50 \%$ when taken with food. Although this prodrug form increases liphophilicity, allowing intestinal absorption, yet it reduces dissolution concurrently. This low dissolution rate may in turn compromise the bioavailability of the drug. In such condition, SD technique using polyvinylpyrrolidone K30 (PVP K30) and polyethylene glycol 4000 (PEG 4000) may 
be employed to improve the dissolution rate and thus indirectly increase the bioavailability of CA.

\section{EXPERIMENTAL}

\section{Materials}

Cefuroxime axetil was obtained as a gift sample from Ipca Laboratories, Mumbai, India. PVP K30 and PEG 4000 were purchased from BASF, Ludwigshafen, Germany.

\section{Preparation of physical mixtures of nitrendipine}

Physical mixtures of CA were prepared by simple blending of $1000 \mathrm{mg}$ CA with a spatula in drug to polymer ratio of $1: 1,1: 3,1: 5$, and $1: 10$ weight ratios. The mixtures were passed through a $500 \mu \mathrm{m}$ mesh sieve for subsequent studies. All weighing were made with weighing balance (AB204-S, Mettler Toledo).

\section{Formulation of solid dispersions of cefuroxime axetil} An amount of $1.5 \mathrm{~g}$ of CA and $1.5 \mathrm{~g}$ of PVP K30, ratio 1:1, were weighed accurately. Then, $50 \mathrm{~mL}$ of methanol were measured using a measuring cylinder and transferred into a $400 \mathrm{~mL}$ beaker. This beaker was left on a hotplate stirrer which had been turn off its temperature while set to stir at $200 \mathrm{rpm}$. The weighed PVP K30 was gradually added into the solution while assuring its complete dissolution with the intermittent addition of methanol. After that, weighed CA was dissolved in the solution again with the intermittent addition of methanol, while $200 \mathrm{~mL}$ of methanol was set as the volume limit to dissolve both CA and PVP K30. The formed solution was left on the stirrer for another additional $5 \mathrm{~min}$ to ensure the homogeneity of the solution. ${ }^{[14]}$

The formed solution was then transferred into a round bottom flask, which later was mounted onto a rotary evaporator, half immersing in a water bath. The warm water bath was set at $40^{\circ} \mathrm{C}$ while the rotary evaporator was set rotating at a moderately high speed. A vacuum aspirator was also connected to the rotary evaporator, which had ice added into it every $2-3 \mathrm{~h}$ to enhance its performance. The set up was run continuously for $12 \mathrm{~h}$ to remove all methanol residues in the round bottom flask forming a dried product. The round bottom flask was then disassembled while a spatula was used to scrap out the formed SD settling onto the flask. The prepared SD was then pulverized in a mortar and pestle without using excessive force. Finally, the end product was passed through a $200 \mu \mathrm{m}$ sieve then kept in an ointment jar storing in a vacuum desiccator. ${ }^{[14]}$

The above protocol was then repeated with each $4.5 \mathrm{~g}, 7.5 \mathrm{~g}$, and $15 \mathrm{~g}$ of PVP K30 replacing $1.5 \mathrm{~g}$ of PVP K30 to form SDs of CA with PVP K30 at 1:3, 1:5, and 1:10 ratios. Again, the protocol was repeated four more times with PEG 4000 in place of PVP $\mathrm{K} 30$ at different ratios; 1:1 (1.5 g of PEG 4000), 1:3 (4.5 g of PEG 4000), 1:5 (7.5 g of PEG 4000), and 1:10 (15 g of PEG 4000). After running all solutions containing CA and PEG 4000 in a rotary evaporator for $12 \mathrm{~h}$, these SD formulations required an additional step such as immersing in an ice water bath for an hour to promote solidification of these formulations, especially the ones with lower PEG 4000 content. ${ }^{[14]}$

\section{Preparation of physical mixtures}

The physical mixtures were prepared by mixing preweighed amounts of mesh. No 100-sieve fractions of CA and carriers in the same proportions as used in SDs. ${ }^{[15]}$

\section{Characterization of solid dispersions Drug content}

An amount of $5 \mathrm{mg}$ of all CA, SDs were weighed and dissolved homogenously with methanol. The solution was suitably diluted and the absorbance was measured at $276 \mathrm{~nm}$. Drug content was calculated using the regression equation. ${ }^{[16]}$

\section{Solubility studies}

A fixed amount of pure CA (5 mg), CA SDs, and CA physical mixtures equivalent to $5 \mathrm{mg}$ of pure $\mathrm{CA}$ were weighed. These weighed samples were sealed tightly and mix with distilled water using an ultrasonicator at $40^{\circ} \mathrm{C}$ for an hour. Following that, these conical tubes were removed and transferred into a shaking incubator, which was set to operate at $37^{\circ} \mathrm{C}, 100 \mathrm{rpm}$ for $24 \mathrm{~h}$. After $24 \mathrm{~h}$, the shaker was switched off while the conical tubes were left in the incubator for another $12 \mathrm{~h}$, incubating at $37^{\circ} \mathrm{C}$. The absorbance values for each sample were measured at $276 \mathrm{~nm}$, in duplicate. The solubility of all CA formulations was compared to that of pure CA while ANOVA was then used to determine the statistical significance. ${ }^{[15-17]}$

\section{Dissolution studies}

Pure CA, SDs, and physical mixtures equivalent to $15 \mathrm{mg}$ of pure CA were tested for their dissolution profile individually in dissolution vessels for a period of $3 \mathrm{~h}$ in hydrochloric acid buffer pH 1.2 and phosphate buffer $\mathrm{pH} 6.8$ separately. During this period, $5 \mathrm{~mL}$ of samples were withdrawn at regular intervals of time and analyzed using Ultraviolet-Vis spectrophotometer at $276 \mathrm{~nm}$. The amount of drug released was calculated using the regression equation obtained from the calibration curve with pure CA. ${ }^{[15-19]}$

\section{Differential scanning calorimetric}

A set of pan and cover, used to form an aluminum crucible, was set on a workstation, which was weighed using an analytical balance. The weight of the aluminum crucible parts and the workstation were nullified. Then, $5 \mathrm{mg}$ of pure CA was then weighed in the aluminum pan. A sharp needle was used to thrust a hole on the aluminum cover. With the aluminum cover on top of the pan, the aluminum crucible was sealed. The crucible was then mounted in a differential scanning calorimetric (DSC) measuring cell while having an empty crucible as a reference. The sample was analyzed from $30^{\circ} \mathrm{C}$ to $210^{\circ} \mathrm{C}, 10^{\circ} \mathrm{C}$ increment $/ \mathrm{min}$. This protocol was then repeated while having all CA formulations, PVP K30, and PEG 4000 in place of pure CA. The graphical results of all formulations were compared to that of pure CA, PVP K30, and PEG 4000. ${ }^{[15,16]}$ 


\section{Fourier transforms infrared spectroscopy spectroscopic analysis}

Fourier transform infrared (FTIR) spectroscopic analysis was performed using FTIR spectroscope (FTIR-8400S, Shimadzu) by the potassium bromide pressed pellet technique. The samples tested included $\mathrm{CA}$, its physical mixture with polymer, and SDs were dried at $35^{\circ} \mathrm{C}$ for $1 / 2$ h to ensure complete removal of moisture. Nitrendipine and dry potassium bromide in the ratio of 1:100 were weighed and ground gently in a clean, dry agate mortar. The sample was transferred to press set, and a pressure of 8 tons was applied for $30 \mathrm{~s}$. The sample was scanned in $4000-400 / \mathrm{cm}$ region at $20 \mathrm{scans} /$ spectrum. ${ }^{[15-18]}$

\section{$X$-ray diffraction studies}

Best samples were selected and sent to be analyzed using an $\mathrm{X}$-ray diffractometer at $30 \mathrm{kV}, 30 \mathrm{~mA}$ over a $2 \theta$ range of $10^{\circ}-100^{\circ}$ by using $\mathrm{Cu} \mathrm{K} \alpha$ radiation. In addition, nickel filter and a step size of 0.01 o were selected. ${ }^{[15,16]}$

\section{Scanning electron microscopy}

Scanning electron microscopy was carried out for the SDs showing maximum release. The surface morphology of the selected binary systems was studied using a Phillips 1500, scanning electron microscope. The powders were previously fixed on a brass stub using double sided adhesive tape and then were made electrically conductive by coating in vacuum, with a thin layer of gold (approximately $300 \AA$ ), for 30 s and at $30 \mathrm{~W}$. The micrographs were taken at an excitation voltage of $15 \mathrm{KV}$ and a magnification of 750 or $5000 \mathrm{X}$.

\section{Kinetic analysis of drug release}

The dissolution profiles of all the SDs were subjected to the kinetic analysis to establish the drug-release mechanism. The release data were fitted to zero order (equation 1), first order (equation 2), matrix (Higuchi model) (equation 3), and HixsonCrowell equations (equation 4) to ascertain the kinetic modeling of drug release. ${ }^{[20,21]}$

$\mathrm{Q}_{\mathrm{t}}=\mathrm{k}_{0} \mathrm{t}$

In $Q_{t}=\operatorname{In} Q_{0}-k_{1} t$

$\mathrm{Q}_{\mathrm{t}}=\mathrm{k}_{\mathrm{H}} \mathrm{t}^{1 / 2}$

$\mathrm{Q}_{0}{ }^{1 / 3}-\mathrm{Qt}^{1 / 3}=\mathrm{k}_{\mathrm{HCt}}$

Where $\mathrm{Q}_{\mathrm{t}}$ is the amount of drug released at time $\mathrm{t}, \mathrm{Q}_{0}$ is the initial amount of drug in the formulation and $\mathrm{k}_{0}, \mathrm{k}_{1}, \mathrm{k}_{\mathrm{H}}$, and $\mathrm{k}_{\mathrm{HC}}$ are release rate constants for zero-order, first-order, Higuchi model, and Hixson-Crowell rate equation.

\section{RESULTS AND DISCUSSION}

All the SDs prepared were found to be fine and free flowing powders. Drug content ranged from 84.2-96.5\%. Low coefficient of variance $(\mathrm{CV})$ values $(<1.0 \%)$ in drug content indicates the reproducibility of the technique employed for the preparation of SDs.

\section{Solubility studies}

The solubility studies of CA and its various formulations are shown in Table 1. Excluding SD of 1:3 and 1:5 ratios, all formulations of PVP K30 had statistical significantly better solubility compared with pure CA. On the other hand, all PEG 4000 physical mixture formulations had lower solubility than pure CA while all PEG 4000 SD formulations had solubility, which were statistical significantly higher than that of pure CA. All SD formulated with PEG 4000 were found having better solubility than pure CA. In addition, the PEG 4000 SD portrayed increment in solubility with an increase in PEG 4000 ratio. However, the maximum increase in solubility relatively to pure CA, shown by CA SD formulated with PEG 4000 at 1:10 ratio, was only 0.74 times, being less than 1 -fold.

\section{Dissolution studies}

Cefuroxime axetil had a poor dissolution profile because it was found agglomerating on top of the dissolution medium which may be due to its hydrophobicity. The increase in dissolution of SD formulated with PVP K30, in this current study was attributed to its good water solubility nature as described by Leuner and Dressman. ${ }^{[23]}$ This nature of PVP K30 increased the solubility and wettability of the SD formulations tremendously, since CA was well dispersed and incorporated in the polymer as proved in the FTIR spectra. In addition, PVP K30 may also prevent crystal growth of CA in SD formulations by forming hydrogen bonds with CA molecules by utilizing the $\mathrm{C}=\mathrm{O}$ bonds of PVP $\mathrm{K} 30$ and N-H bonds of CA as suggested by Jun et al. ${ }^{[22]}$ Leuner and Dressman. ${ }^{[23]}$ This would thus promote amorphization and particle size reduction, not forgetting increasing the interfacial surface area for dissolution. ${ }^{[15,16]}$ Yet, this interaction was not supported by the inconclusive spectra of PVP K30 SD

\begin{tabular}{lccc} 
Table 1: Solubility of CA and its formulations \\
\hline Formulations & $\begin{array}{c}\text { Percentage } \\
\text { solubility }\end{array}$ & $\begin{array}{c}\text { Relative } \\
\text { solubility } \\
\text { to CA }\end{array}$ & $\begin{array}{c}\text { Relative } \\
\text { solubility to } \\
\text { CA (\%) }\end{array}$ \\
\hline CA & 68.91 & 1.00 & 1.00 \\
CA + PVP_PM 1:1 & $73.40^{*}$ & 1.07 & 6.51 \\
CA + PVP_PM 1:3 & $86.17^{*}$ & 1.25 & 25.04 \\
CA + PVP_PM 1:5 & $83.72^{*}$ & 1.21 & 21.49 \\
CA + PVP_PM 1:10 & $76.79^{*}$ & 1.11 & 11.44 \\
CA + PVP_SD 1:1 & $77.47^{*}$ & 1.12 & 12.42 \\
CA + PVP_SD 1:3 & 61.42 & 0.89 & -10.88 \\
CA + PVP_SD 1:5 & 62.41 & 0.91 & -9.44 \\
CA + PVP_SD 1:10 & $95.68^{*}$ & 1.39 & 38.84 \\
CA + PEG 4000_PM 1:1 & 38.61 & 0.56 & -43.97 \\
CA + PEG 4000_PM 1:3 & 56.55 & 0.82 & -17.94 \\
CA + PEG 4000_PM 1:5 & 39.84 & 0.58 & -42.19 \\
CA + PEG 4000_PM 1:10 & 39.29 & 0.57 & -42.98 \\
CA + PEG 4000_SD 1:3 & $111.08^{*}$ & 1.61 & 61.18 \\
CA + PEG 4000_SD 1:5 & $112.27^{*}$ & 1.63 & 62.91 \\
CA + PEG 4000_SD 1:10 & $119.97^{*}$ & 1.74 & 74.09 \\
\hline *The mean difference is significant at the o.05 level. Dunnett $t$-test was used while \\
CA was taken as the control. CA: Cefuroxime axetil, PVP: Polyvinylpyrrolidone, \\
PM: Physical mixtures, SD: Solid dispersions, PEG: Polyethylene glycol
\end{tabular}


formulations in this current study. Another probable reason for this increase in dissolution profile of CA SD may be due to the formation of $\beta$ crystalline form, documented having a higher bioavailablity, which was suggested present in FTIR spectra. Similarly to PVP K30, PEG 4000 increased its SD's dissolution rate by increasing their solubility and wettability as described by Leuner and Dressman. ${ }^{[23]}$ All PEGs are documented as having good water solubility, with the lower molecular weight ones, including PEG 4000, having a relatively better solubility. ${ }^{[16]}$ PEG 4000 may also promote amorphization, like PVP K30, causing a reduction in particle size and increasing interfacial surface area required for dissolution.

As explained by Leuner and Dressman, ${ }^{[23]}$ the dissolution rate of SD formulations increased with both PVP K30 and PEG 4000 ratio among formulations, because it was found that the dissolution rate is only dependent solely on the carrier when there is a low drug to carrier ratio. Moreover, reduction of crystallinity generally increases with increasing carrier ratio thus increasing the solubility and release rate of the SD. In case, where the very high carrier ratio was used such as 1:10 ratio as done in this current study, a metastable critical mixture at its saturation point may be formed. Thus, it would have maximum solubility.

Dissolution profile of CA SD formulated with PEG 4000 at $1: 1$ ratio was not conducted. This was due to the fact that $P E G$ 4000 SD formulations with low PEG 4000 content appeared in a sol form which had a sticky consistency, with the worst being that of $1: 1$ ratio. This phenomenon may be due to PEG 4000 having a relatively too low molecular weight making it unsuitable for formulation with CA, alike to many other drugs reported by Leuner and Dressman. ${ }^{[23]}$ CA may also had caused this phenomenon by inducing plasticity onto PEG 4000, as suggested by Leuner and Dressman, since it was found that the higher PEG 4000 ratio used, the less sticky the formulation became. ${ }^{[23]}$ The dissolution pattern for pure CA and its prepared SD with different carriers is shown in Tables 2 and 3, Figures 1-4.

Table 2: Dissolution rate of $\mathrm{CA}$ and its formulations in $\mathrm{pH} 1.2$ hydrochloric acid buffer

\begin{tabular}{|c|c|c|c|c|}
\hline \multirow[t]{2}{*}{ Formulations } & \multicolumn{4}{|c|}{ Percentage dissolution in pH 1.2 hydrochloric acid buffer } \\
\hline & $10 \mathrm{~min}$ & $15 \mathrm{~min}$ & $30 \mathrm{~min}$ & $40 \mathrm{~min}$ \\
\hline CA & $20.47 \pm 0.63$ & $32.56 \pm 5.44$ & $39.54 \pm 3.67$ & $50.27 \pm 13.38$ \\
\hline CA + PVP K30_PM 1:1 & $39.55 \pm 3.41^{*}$ & $53.61 \pm 3.68^{*}$ & $65.18 \pm 4.44^{*}$ & $67.66^{*} \pm 4.36$ \\
\hline CA + PVP K30 PM 1:3 & $61.88 \pm 2.22^{*}$ & $67.76 \pm 4.86^{*}$ & $69.44 \pm 4.97^{*}$ & $70.26 \pm 5.22^{*}$ \\
\hline CA + PVP K30_PM 1:5 & $71.27 \pm 2.53^{*}$ & $77.06 \pm 0.99^{*}$ & $75.98 \pm 1.15^{\star}$ & $73.98 \pm 1.78^{*}$ \\
\hline CA + PVP K30_PM 1:10 & $72.90 \pm 1.93^{*}$ & $84.49 \pm 5.16^{*}$ & $85.91 \pm 1.14^{*}$ & $85.15 \pm 1.94^{*}$ \\
\hline CA + PVP K30_SD 1:1 & $32.45 \pm 1.09^{*}$ & $41.11 \pm 0.81^{*}$ & $57.51 \pm 2.46^{\star}$ & $62.53 \pm 3.28 *$ \\
\hline CA + PVP K30_SD 1:3 & $95.73 \pm 1.76^{*}$ & $96.77 \pm 0.61^{*}$ & $95.98 \pm 1.28^{*}$ & $95.75 \pm 0.52^{*}$ \\
\hline CA + PVP K30_SD 1:5 & $99.31 \pm 0.76^{*}$ & $99.46 \pm 0.70^{*}$ & $97.97 \pm 1.55^{\star}$ & $96.22 \pm 2.75^{\star}$ \\
\hline CA + PVP K30_SD 1:10 & $133.15 \pm 2.43^{*}$ & $134.12 \pm 2.63^{*}$ & $132.01 \pm 2.76^{*}$ & $127.10 \pm 3.43^{*}$ \\
\hline $\mathrm{CA}+\mathrm{PEG} 4000$ PM $1: 1$ & $22.13 \pm 2.26$ & $27.72 \pm 2.71$ & $41.06 \pm 4.46$ & $49.52 \pm 3.81$ \\
\hline CA + PEG 4000-PM 1:3 & $41.77 \pm 14.54^{*}$ & $39.70 \pm 3.06^{*}$ & $48.50 \pm 7.18^{*}$ & $53.24 \pm 7.14$ \\
\hline CA + PEG 4000_PM 1:5 & $46.86 \pm 0.78^{\star}$ & $48.91 \pm 0.31 *$ & $51.79 \pm 1.32^{*}$ & $52.39 \pm 2.83$ \\
\hline CA + PEG 4000_PM 1:10 & $70.72 \pm 9.09^{\star}$ & $62.26 \pm 0.13^{*}$ & $62.56 \pm 1.32^{\star}$ & $61.86 \pm 1.93^{*}$ \\
\hline CA + PEG 4000_SD 1:3 & $92.42 \pm 2.81^{*}$ & $92.45 \pm 4.66^{*}$ & $92.04 \pm 4.60^{*}$ & $91.67 \pm 4.40^{*}$ \\
\hline CA + PEG 4000_SD 1:5 & $108.90 \pm 2.00^{*}$ & $105.75 \pm 0.91^{*}$ & $104.36 \pm 0.72^{*}$ & $103.65 \pm 0.91^{*}$ \\
\hline CA + PEG 4000_SD 1:10 & $104.08 \pm 0.27^{*}$ & $103.62 \pm 0.38^{*}$ & $102.50 \pm 0.79^{*}$ & $101.39 \pm 0.65^{*}$ \\
\hline
\end{tabular}

*The mean difference is significant at the .05 level. Dunnett $t$-test was used while CFA was taken as the control. CFA: Confirmatory factor analysis, CA: Cefuroxime axetil, PVP: Polyvinylpyrrolidone, PM: Physical mixtures, SD: Solid dispersions, PEG: Polyethylene glycol

Table 3: Dissolution rate of CA and its formulations in $\mathrm{pH} 6.8$ phosphate buffer

\begin{tabular}{|c|c|c|c|c|}
\hline \multirow[t]{2}{*}{ Formulations } & \multicolumn{4}{|c|}{ Dissolution rate in $\mathrm{pH} 6.8$ phosphate buffer } \\
\hline & $10 \mathrm{~min}$ & $15 \mathrm{~min}$ & $30 \mathrm{~min}$ & $40 \mathrm{~min}$ \\
\hline $\mathrm{CA}$ & $23.03 \pm 7.01$ & $33.03 \pm 6.13$ & $43.85 \pm 7.97$ & $45.06 \pm 8.37$ \\
\hline CA + PVP K30_PM 1:1 & $41.41 \pm 6.80$ & $51.95 \pm 6.84^{*}$ & $63.17 \pm 4.50$ & $66.46 \pm 3.26 *$ \\
\hline CA + PVP K30_PM 1:3 & $66.59 \pm 2.84^{*}$ & $72.77 \pm 2.47^{*}$ & $74.27 \pm 2.47$ & $73.71 \pm 2.40$ * \\
\hline CA + PVP K30_PM 1:5 & $71.96 \pm 1.69^{*}$ & $79.15 \pm 1.46^{*}$ & $80.77 \pm 0.84^{*}$ & $80.37 \pm 0.93^{*}$ \\
\hline CA + PVP K30_PM 1:10 & $72.32 \pm 2.56^{*}$ & $79.65 \pm 2.63^{*}$ & $80.49 \pm 2.54^{*}$ & $78.01 \pm 2.72^{*}$ \\
\hline CA + PVP K30_SD 1:1 & $37.69 \pm 3.70$ & $45.36 \pm 4.32^{*}$ & $56.61 \pm 3.40$ & $61.14 \pm 3.56^{*}$ \\
\hline CA + PVP K30_SD 1:3 & $87.62 \pm 1.23^{*}$ & $88.26 \pm 1.88^{*}$ & $87.13 \pm 1.75^{\star}$ & $86.96 \pm 1.65^{\star}$ \\
\hline CA + PVP K30_SD 1:5 & $93.52 \pm 2.71^{*}$ & $93.59 \pm 2.92^{*}$ & $91.75 \pm 1.61^{*}$ & $91.29 \pm 1.73^{*}$ \\
\hline CA + PVP K30_SD 1:10 & $128.48 \pm 2.16^{*}$ & $127.21 \pm 1.41^{*}$ & $125.29 \pm 0.97^{\star}$ & $124.83 \pm 1.02$ \\
\hline CA + PEG 4000_PM 1:1 & $29.51 \pm 3.17$ & $38.41 \pm 1.96$ & $54.05 \pm 2.79$ & $59.55 \pm 4.74$ * \\
\hline CA + PEG 4000_PM 1:3 & $32.58 \pm 0.36$ & $41.74 \pm 2.53^{*}$ & $59.04 \pm 2.21$ & $61.04 \pm 1.92 *$ \\
\hline CA + PEG 4000_PM 1:5 & $36.12 \pm 1.92$ & $39.68 \pm 1.37$ & $44.09 \pm 1.86$ & $45.40 \pm 2.22$ \\
\hline CA + PEG 4000_PM 1:10 & $47.44 \pm 2.53^{*}$ & $48.78 \pm 1.91^{*}$ & $49.52 \pm 1.81$ & $49.36 \pm 1.66$ \\
\hline CA + PEG 4000_SD 1:3 & $94.82 \pm 0.35^{\star}$ & $94.88 \pm 2.57^{*}$ & $94.76 \pm 2.23^{*}$ & $94.64 \pm 2.04^{*}$ \\
\hline CA + PEG 4000_SD 1:5 & $102.64 \pm 3.29^{*}$ & $102.94 \pm 3.08^{*}$ & $102.52 \pm 2.86^{*}$ & $102.36 \pm 3.25$ \\
\hline CA + PEG 4000_SD 1:10 & $99.79 \pm 0.43^{*}$ & $100.15 \pm 0.68^{*}$ & $99.82 \pm 0.58^{*}$ & $99.12 \pm 0.89^{*}$ \\
\hline
\end{tabular}

*The mean difference is significant at the .05 level. Dunnett $t$-test was used while CFA was taken as the control. CFA: Confirmatory factor analysis, CA: Cefuroxime axetil, PVP: Polyvinylpyrrolidone, PM: Physical mixtures, SD: Solid dispersions, PEG: Polyethylene glycol 


\section{Differential scanning calorimetric analysis}

The thermogram of pure CA was able to prove that the pure CA used in this study existed as polymorphs. ${ }^{[24]}$ As found by Crisp et al. ${ }^{[24]}$ and Sasinowska et al., ${ }^{[25]} \mathrm{CA}$ exists as three polymorphs, namely $\mathrm{A}^{\mathrm{I}} \mathrm{A}^{\mathrm{II}}$ and $\mathrm{K}^{[25]} \mathrm{A}^{\mathrm{I}}$ and $\mathrm{A}^{\mathrm{II}}$ polymorphs are known to exist as amorphous forms, usually showing endothermic bands around $94^{\circ} \mathrm{C}$ and 125 ${ }^{\circ} \mathrm{C}$ in thermograms, while $\mathrm{K}$ polymorph exists as crystalline form with a melting point of $181^{\circ} \mathrm{C}$. Referring to the thermogram of CA, it was found that the CA raw material used in this study contained higher proportions of $\mathrm{A}^{\mathrm{I}}$ and $\mathrm{K}$ polymorph and lower proportions of $\mathrm{A}^{\mathrm{II}}$ polymorphs. Referring to the DSC thermogram of pure PVP $\mathrm{K} 30$, a very broad endothermic band around $89^{\circ} \mathrm{C}$ was found which was caused by its loss of water in the calorimetric analysis.

The DSC thermograms of all physical mixtures formulated with CA and PVP K30 proved the presence of $\mathrm{A}^{\mathrm{I}}$ and $\mathrm{K}$ polymorph, signifying that CA existed as both amorphous and crystalline form in physical mixtures. The thermograms also showed that CA was better dispersed among physical mixtures of higher PVP K30 ratio through the shrinking of CA peaks with increasing PVP K30 ratio. Apart from that, these thermograms of physical mixtures each contained large endothermic bands which signified the presence of water, similarly to the thermogram of pure PVP K30.

On the contrary, all DSC thermograms of PVP K30 SD only verified the presence of water and showed a minor endothermic band appearing at the range of $145-155^{\circ} \mathrm{C}$. This band may be evidence of the presence of $\mathrm{K}$ polymorph which had been shifted. It may also signify the presence of another crystalline polymorph of CA which was newly discovered and found to have a better oral

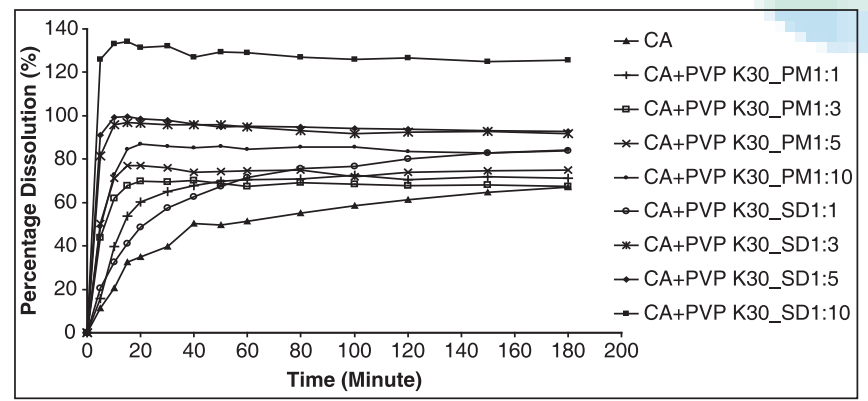

Figure 1: Dissolution profile of cefuroxime axetil and its polyvinylpyrrolidone $\mathrm{K} 30$ formulations in $\mathrm{pH} 1.2$, hydrochloric acid buffer

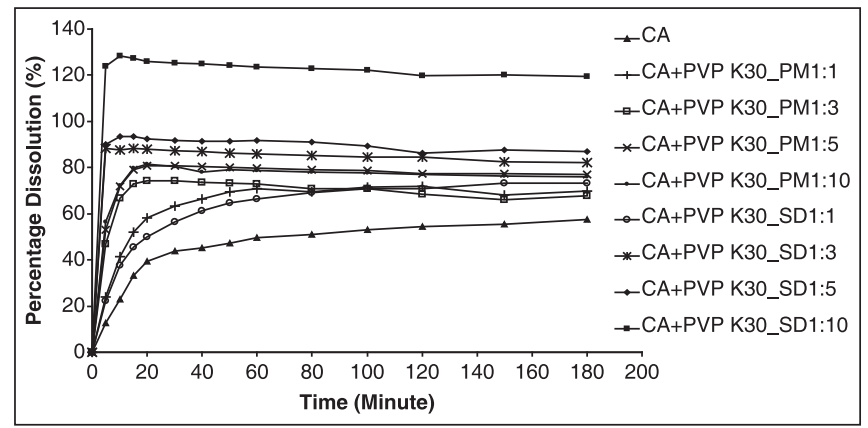

Figure 3: Dissolution profile of cefuroxime axetil and its polyvinylpyrrolidone $\mathrm{K} 30$ formulations in $\mathrm{pH} 6.8$, phosphate buffer bioavailability. The DSC thermograms of CA, various physical mixtures and SD are given in Figures 5 and 6.

Looking at the thermograms of all formulations made with PEG 4000 , despite being SD or physical mixture, they only contained a peak corresponding to PEG 4000, signifying that CA was homogenously dispersed in an amorphous state.

\section{Fourier transform infrared spectroscopy analysis}

As reported by Jun et al. ${ }^{[22]}$ and World Property Intellectual Organization, ${ }^{[26]}$ the spectrum of pure CA showed the presence of stretching bonds between N-H and N-H $(3483 / \mathrm{cm}$, $3363 / \mathrm{cm}$, and $3313 / \mathrm{cm})$. Peaks are representing $\mathrm{C}=\mathrm{O}$ of $\beta$ lactam, ester, carbamyl, and amido were also spotted in the spectrum $(1786 / \mathrm{cm}, 1736 / \mathrm{cm}, 1686 / \mathrm{cm}$, and $1543 / \mathrm{cm})$. Referring to the spectrum of PVP K30, its functional groups with its corresponding peaks were summarized as follow: O-H $(3450 / \mathrm{cm}), \mathrm{C}-\mathrm{H}(2924 / \mathrm{cm}), \mathrm{C}=\mathrm{O}(1655 / \mathrm{cm})$, and C-O-C $(1022 / \mathrm{cm})$. In addition, the spectrum of PVP K30 also had a broad band caused by the presence of water.

Similarly, the spectra of all formulations between CA and PVP $\mathrm{K} 30$ contained peaks representing ester, carbamyl, and amido while the peak of $\beta$ lactam was shifted. However, this shift was concluded as minor and insignificant. This intense similarity in spectra of both CA and its formulations may suggest that the molecular structure of CA in all formulations, despite being solid dispersions or physical mixtures, was still intact. The absence of peak shift among carbonyl group may suggest that there was no significant chemical interaction between PVP K30 and CA thus

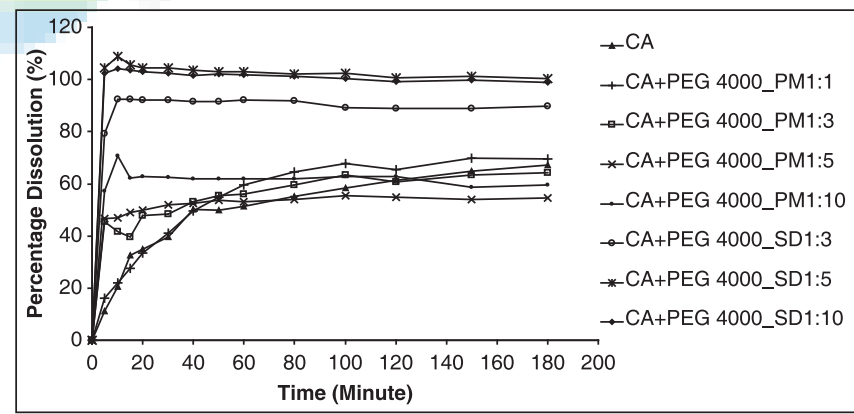

Figure 2: Dissolution profile of cefuroxime axetil and its polyethylene glycol 4000 formulations in $\mathrm{pH} 1.2$, hydrochloric acid buffer

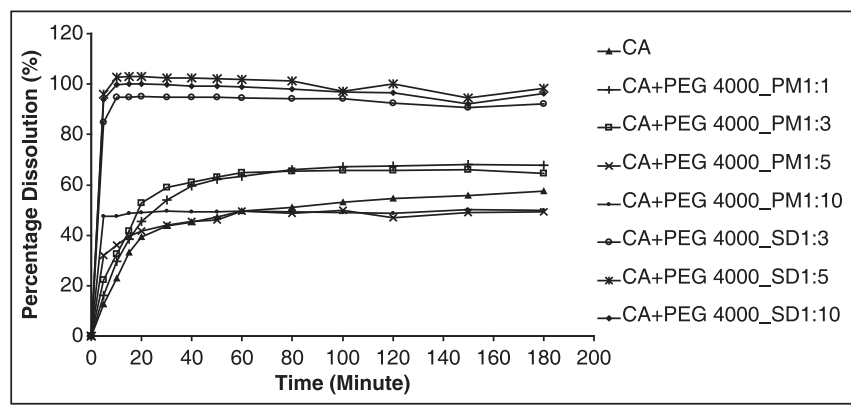

Figure 4: Dissolution profile of cefuroxime axetil and its polyethylene glycol 4000 formulations in $\mathrm{pH}$ 6.8, phosphate buffer 
the antibacterial effect of CA may be proved preserved as well. In addition, the result for $\mathrm{N}-\mathrm{H}$ peaks was inconclusive due to the fact that both CA and PVP K30 had peaks around this region. Moreover, there existed a broad band probably arising due to the presence of water, masking the intensity of most peaks. ${ }^{[22,26]}$

All PEG 4000 formulations, both SD, and physical mixtures were found exhibiting characteristic peaks of PEG 4000. Due to too small a transmittance, $\mathrm{C}=\mathrm{O}$ peaks of $\mathrm{CA}$ in these physical mixtures could not had their wavelengths documented excluding

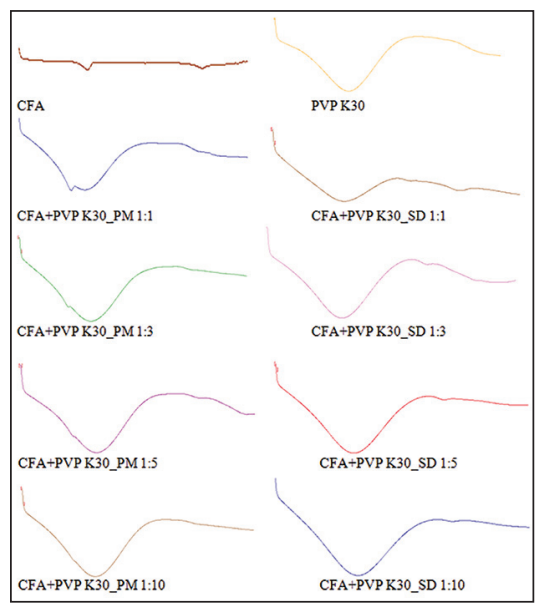

Figure 5: Differential scanning calorimetric thermograms of cefuroxime axetil, polyvinylpyrrolidone $\mathrm{K} 30$, and their formulations

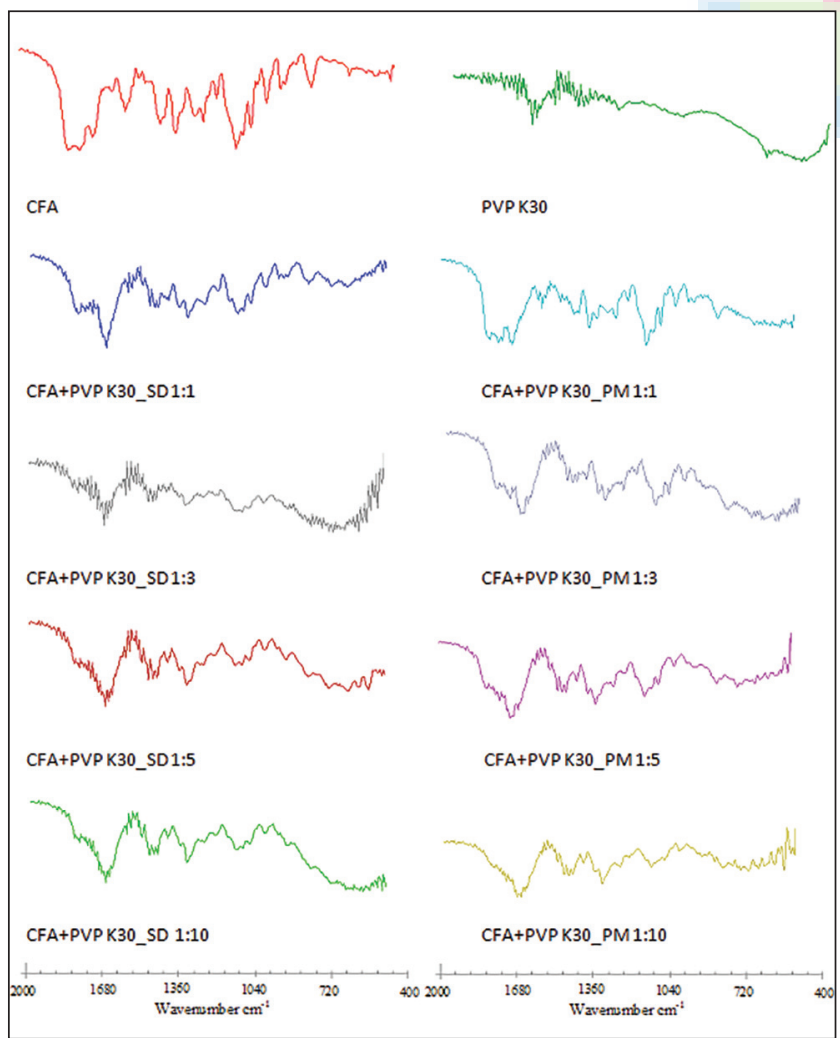

Figure 7: Fourier transform infrared spectra of cefuroxime axetil, polyvinylpyrrolidone $\mathrm{K} 30$, and their formulations that of $\beta$ lactam. Spectra of CA SD formulated with PEG 4000, however, portrayed the appearance of new peaks in the $\mathrm{C}=\mathrm{O}$ region, while $\mathrm{C}=\mathrm{O}$ peaks of $\mathrm{CA}$ were not found. This may indicate the occurrence of peak shifting, suggesting the occurrence of interactions between CA and PEG 4000 ${ }^{[22,24]}$ [Figures 7 and 8].

\section{Powder X-ray diffraction studies}

The diffractogram of pure CA was of a halo pattern, being broad and having diffused maxima, depicting that of an amorphous

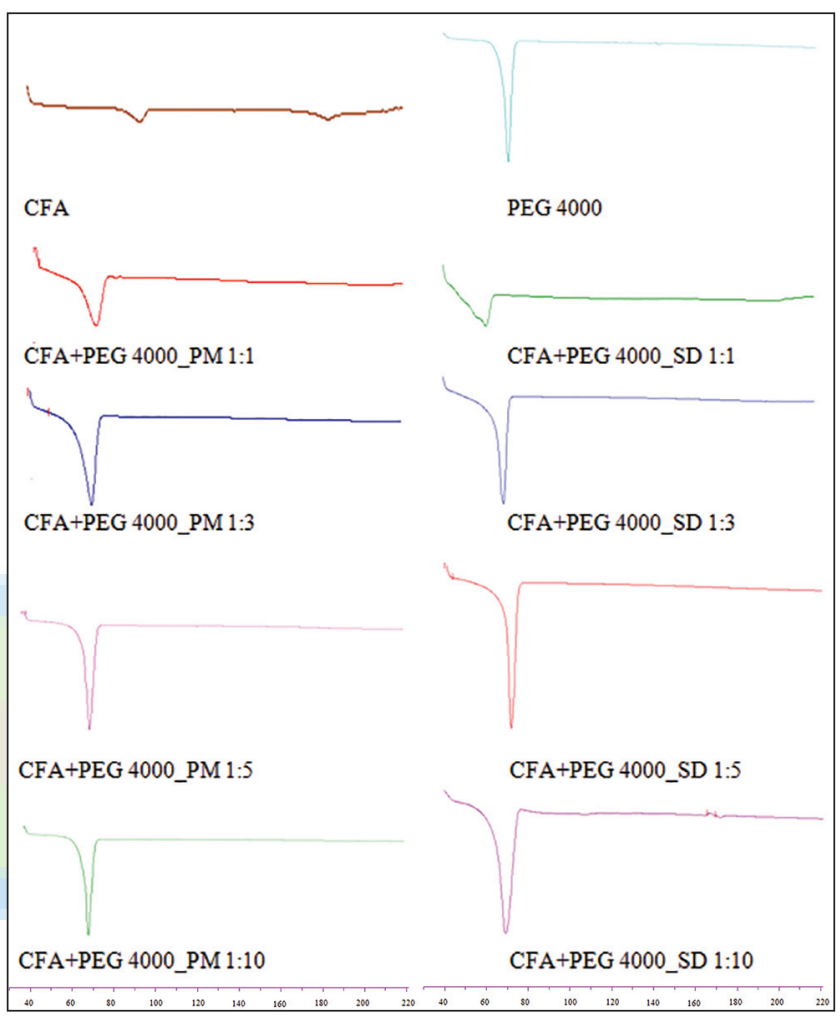

Figure 6: Differential scanning calorimetric thermograms of cefuroxime axetil, polyethylene glycol 4000 , and their formulations

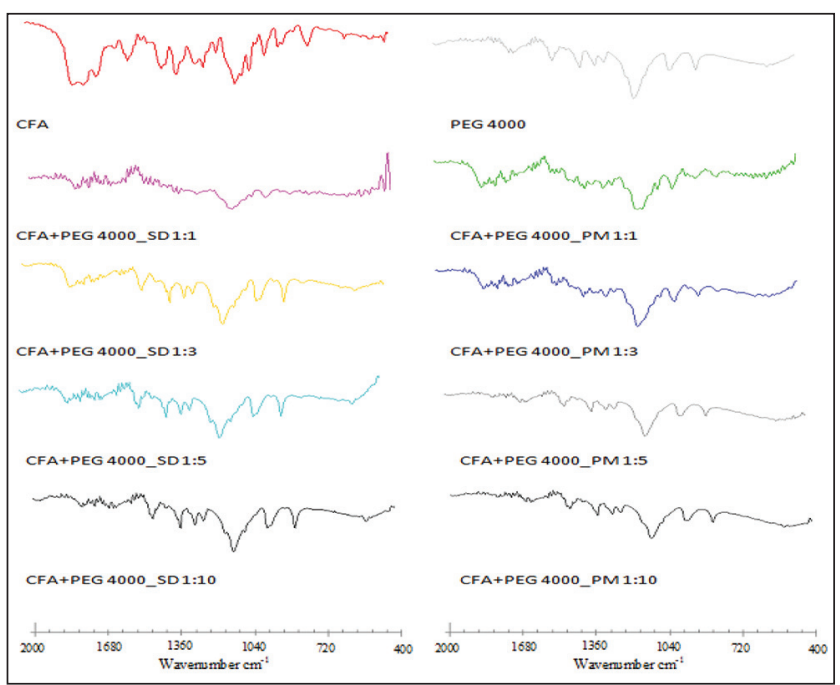

Figure 8: Fourier transform infrared spectra of cefuroxime axetil, polyethylene glycol 4000 , and their formulations 
compound. This was found somewhat in agreement with the results of DSC, representing the presence of A polymorphs yet the presence of $\mathrm{K}$ polymorph was nowhere to be seen in the diffractograms. However, although Zhang et al. ${ }^{[27]}$ and Jun et al. ${ }^{[22]}$ reported that their drugs were of polymorphs as well, being in both amorphous and crystalline form, yet their reported diffractograms of pure CA showed sharp diffraction peaks belonging to $\mathrm{K}$ polymorph, contradictory to this current research. The diffractogram of PVP K30 was also absent of sharp peaks, being broad with two diffused maxima. This confirmed that PVP $\mathrm{K} 30$ appeared in an amorphous form. The diffractograms of both SD and physical mixture with CA to PVP K30 ratio of 1:10 was illustrating a similar pattern as that of pure PVP K30; thus, these formulations were confirmed to exist in amorphous form as well.

The diffractogram of PEG 4000 confirmed that PEG 4000 exists as a somewhat crystalline form by having sharp diffraction peaks, matching the results found by Martinez-Oharriz et al ${ }^{[28]}$ The diffractograms of both SD and physical mixture with CA to PEG 4000 ratio of 1:10 were found similar to PEG 4000, while absent of any sharp diffraction peaks that might be CA origin. Thus, CA was concluded to be in an amorphous form while PEG 4000 in a somewhat crystalline form in these formulations [Figures 9 and 10].

\section{Scanning electron microscopy}

Cefuroxime axetil appeared as irregular shaped crystals. The original morphology of all other binary systems had disappeared and it was not possible to differentiate between the two components. All the binary systems appeared as agglomerates exhibiting the presence of a homogeneous solid phase of amorphous nature. Existence of a single phase is also responsible for the enhanced drug dissolution in comparison to pure CA [Figure 11].

\section{Kinetic analysis of drug release}

The kinetics of in vitro release of the best formulations of were carried out. The release of drug from all formulations was observed to follow the first order release kinetics, since the correlation coefficient $\left(\mathrm{R}^{2}\right)$ for the first order was higher in comparison to zero order release. The results were in agreement with the previous investigations performed by Dua et. al, ${ }^{[20,29]}$ El-Maradny et al. ${ }^{[27]}$

The data were further subjected to Higuchi equation and Hixson-Crowell cube root law. A higher correlation, as indicated by $\mathrm{R}^{2}$ was observed for the Higuchi matrix release kinetics in all the selected formulations suggesting the diffusion as a probable prominent mechanism of drug release. ${ }^{[27]}$ In diffusion, the rate of dissolution of drug particles within the matrix must be much faster than that of the diffusion rate of drug leaving the matrix.

\section{CONCLUSIONS}

This study clearly shows that the addition of various hydrophilic carriers like PVP K30 and PEG 4000 to CA improves its dissolution

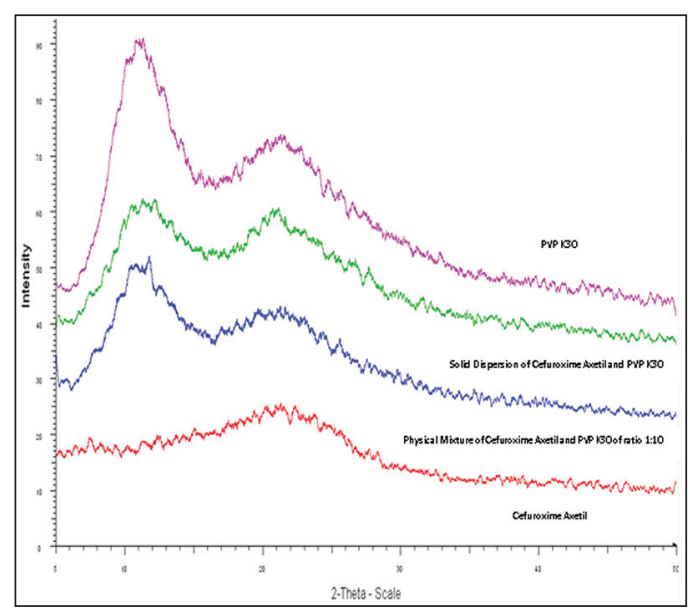

Figure 9: Powder X-ray diffractograms of cefuroxime axetil, polyvinylpyrrolidone $\mathrm{K} 30$, and their formulations

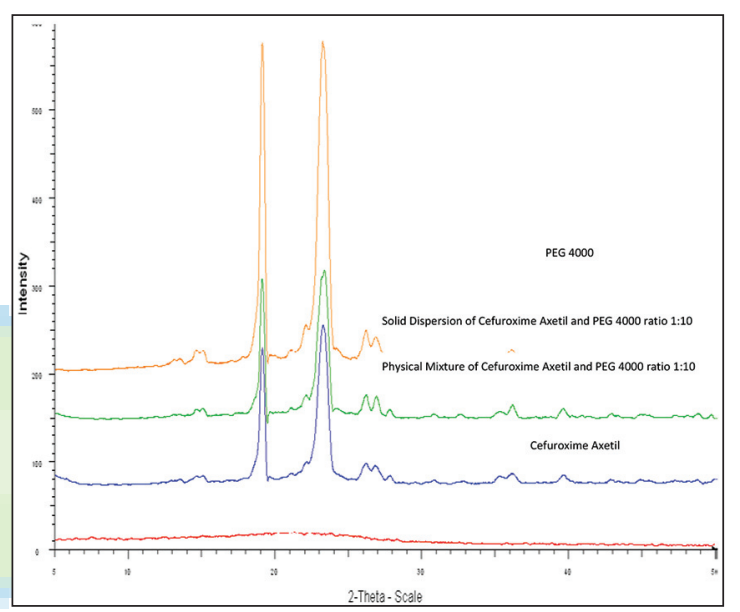

Figure 10: Powder X-ray diffractograms of cefuroxime axetil, polyethylene glycol 4000 , and their formulations

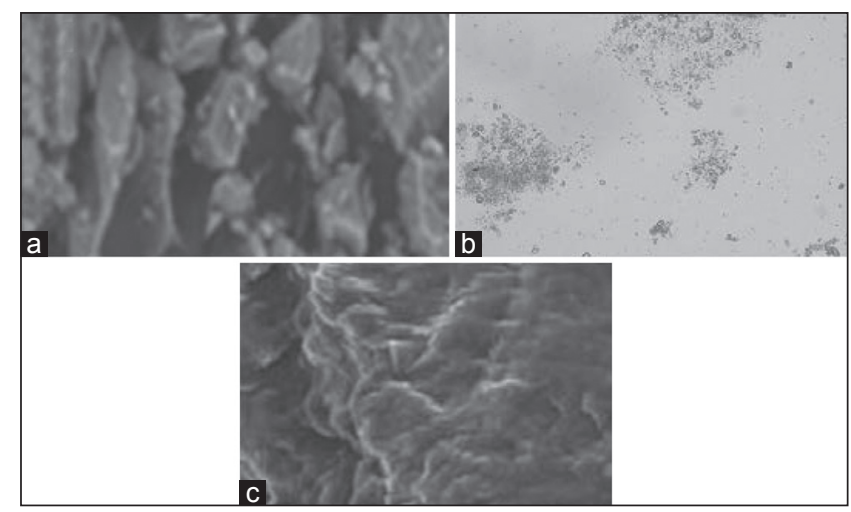

Figure 11: Scanning Electron Micrograph of (a) cefuroxime axetil (CA); (b) solid dispersion (SD) of CA, and polyvinylpyrrolidone K30; and (c) SD of CA and polyethylene glycol 4000

rate. Further, all the SD performed better than the corresponding physical mixtures. The higher dissolution rate of SD may be due to increase wettability, solubility, and reduction in particle size. DSC thermograms of the physical mixture and SD indicated complete miscibility of the drug in melted carrier. Amorphous nature of 
the drug in SD was confirmed by X-ray diffractograms. Results from FTIR spectroscopy concluded that there was no well-defined interaction between CA and carriers employed in the preparation of SD. The SD of CA with PVP K30 and PEG 4000, lends an ample credence for better therapeutic efficacy.

\section{REFERENCES}

1. Hite M, Turner SF. Pharmaceutical Manufacturing and Packing Sourcer, Part 1. Oral Deliv of Poorly Soluble Drugs; Summer; 2003. p. 29-1. Available from: http://www. scolr.com/lit/ PMPS_2003_1.pdf. [Last accessed 2012 Oct 1].

2. Goldberg $A H$, Gibaldi M, Kanig JL. Increasing dissolution rates and gastrointestinal absorption of drugs via solid solutions and eutectic mixtures. I. Theoretical considerations and discussion of the literature. J Pharm Sci 1965;54:1145-8.

3. Goldberg AH, Gibaldi M, Kanig JL. Increasing dissolution rates and gastrointestinal absorption of drugs via solid solutions and eutectic mixtures - II - Experimental evaluation of eutectic mixture: Urea-acetaminophen system. J Pharm Sci 1966;55:482-7.

4. Goldberg AH, Gibaldi M, Kanig JL, Mayersohn M. Increasing dissolution rates and gastrointestinal absorption of drugs via solid solutions and eutectic mixtures. IV. Chloramphenicol urea system. J Pharm Sci 1966;55:581-3.

5. Hoerter D, Dressman JB. Influence of physicochemical properties on dissolution of drugs in the gastrointestinal tract. Adv Drug Deliv Res 1997;25:3-14.

6. Hancock BC, Zografi G. Characteristics and significance of the amorphous state in pharmaceutical systems. J Pharm Sci 1997;86:1-12.

7. Loftsson T, Brewster ME. Pharmaceutical applications of cyclodextrins 1. Drug solubilization and stabilization. J Pharm Sci 1996;85:1017-25.

8. Chiou WL, Riegelman S. Pharmaceutical applications of solid dispersion systems. J Pharm Sci 1971;60:1281-302.

9. Sekiguchi K, Obi N. Studies on absorption of eutectic mixtures - I. A comparison of the behavior of eutectic mixture of sulphathiazole and that of ordinary sulphathiazole in man. Chem Pharm Bull 1961;9:866-72.

10. Tachibana T, Nakamura A. A method for preparing an aqueous colloidal dispersion of organic material by using water soluble polymers: Dispersion of beta-carotene by polyvinylpyrrolidone. Kolloid Z Polym 1965;203:130-3.

11. Swarbrick J, Boylon J. Encyclopedia of Pharmaceutical Technology. $2^{\text {nd }}$ ed., Vol. I. New York: Marcel Dekker; 2002. p. 641-8.

12. Dellamonica P. Cefuroxime axetil. Int J Antimicrob Agents 1994;4:23-36.

13. Pichichero ME. Use of selected cephalosporins in penicillinallergic patients: A paradigm shift. Diagn Microbiol Infect Dis 2007; $57: 13$ S-8.

14. Sethia S, Squillante E. Solid dispersion of carbamazepine in PVP K30 by conventional solvent evaporation and supercritical methods. Int J Pharm 2004;272:1-10.
15. Dua K, Pabreja K, Ramana MV, Bukhari NI. Preparation, characterization and in vitro evaluation of aceclofenac PVP-solid dispersions. J Dispers Sci Technol 2011;32:1151-7.

16. Dua K, Pabreja K, Ramana MV. Enhancement of dissolution behaviour of aceclofenac by complexation with $\beta$-cyclodextrincholine dichloride. J Dispers Sci Technol 2011;32:1477-84.

17. Lim YL, Huan WS, Adhiyaman R, Gorajana A, Dua K, Garg S. Studies on development and dissolution rate of piroxicam using crystal modifications. Acta Pharm 2011;61:391-402.

18. Ramana MV, Chaudhari AD, Himaja M, Satyanaryana D, Dua K. An approach to minimize pseudomembranous colitis caused by clindamycin through liposomal formulation. Indian J Pharm Sci 2007;4:392-3.

19. Rowe RC, Sheskey P, Owen SC. Handbook of Pharmaceutical Excipients. $5^{\text {th }}$ ed. London: Pharmaceutical Press; 2005. p. 34-6.

20. Dua K, Pabreja K, Ramana MV, Lather V. Dissolution behaviour of ß-cyclodextrin molecular inclusion complexes of aceclofenac.J Pharm Bioallied Sci 2011;3:417-25.

21. Costa P, Sousa Lobo JM. Modeling and comparison of dissolution profiles. Eur J Pharm Sci 2001;13:123-33.

22. Jun SW, Kim MS, Jo GH, Lee S, Woo JS, Park JS, et al. Cefuroxime axetil solid dispersions prepared using solution enhanced dispersion by supercritical fluids. J Pharm Pharmacol 2005;57:1529-37.

23. Leuner C, Dressman J. Improving drug solubility for oral delivery using solid dispersions. Eur J Pharm Biopharm 2000;50:47-60.

24. Crisp HA, Clayton JC, Elliot LG, Wilson EM. Preparation of Highly Pure, Substantially Amorphous Form of Cefuroxime Axetil: US Patent 4820833; 1985.

25. Sasinowska M, Winiewska I, Gumulka W, Oszczapowicz I, Szelachowska M, Interewiez B. Esters of Cephalosporins, Part I. Permeability of cefuroxime liberated from its 1-acetoxyethyl ester through biological membranes; influence of the form and size of the ester particles. Acta Pol Pharm 1995;52:397-401.

26. World Intellectual Property Organization. (WO/1998/043980) A Process for Preparing an Amorphous Cefuroxime Axetil Having a Low Melting Point. Available from: http://www.wipo.int/pctdb/en/ wo.jsp?IA=KR1997000194\&DISPLAY=DESC. [Last accessed on 2008 Nov 12].

27. Zhang JY, Shen ZG, Zhong J, Hu TT, Chen JF, Zhong QM, et al. Preparation of amorphous cefuroxime axetil nanoparticles by controlled nanoprecipitation method without surfactants. Pharm Nanotechnol 2006;323:1-2.

28. Martínez-Ohárriz MC, Martín C, Goñi MM, RodríguezEspinosa C, Tros-llarduya MC, Zornoza A. Influence of polyethylene glycol 4000 on the polymorphic forms of diflunisal. Eur J Pharm Sci 1999;8:127-32.

29. El-Maradny HA, Mortada SA, Kamel OA, Hikal AH. Characterization of ternary complexes of meloxicam-HPbetaCD and PVP or L-arginine prepared by the spray-drying technique. Acta Pharm 2008;58:455-66.

How to cite this article: Gorajana A, Rajendran A, Yew LM, Dua K. Preparation and characterization of cefuroxime axetil solid dispersions using hydrophilic carriers. Int J Pharma Investig 2015;5:171-8.

Source of Support: Nil. Conflicts of Interest: None declared. 